\title{
The Hole Argument Against Everything (uncut)
}

\author{
Joshua Norton
}

University of California, Irvine

Logic and Philosophy of Science

\section{Introduction}

Note: the following is a slightly expanded version of the accepted paper. Almost all additions occur in section $\S 4$.

The Hole Argument can be extended to exclude everything. I will argue that there is nothing in the metaphysical commitment of a substantival manifold which makes it especially susceptible to the Hole Argument; other objects are just as susceptible to its terrors. These casualties of the hole demonstrate how critically the Hole Argument hinges on our notion of determinism and not on the diffeomorphic freedom of general relativity (GR). Just as Earman and Norton [11] argue that we should not let our metaphysics run roughshod over the structure of our physical theories, so I will argue that, in particular, we should not uncritically allow our metaphysics to dictate what our physical theories must determine. The central conviction which drives the 
arguments of this paper is that deterministic theories are not required to determine for future moments what they cannot determine for any present or past moments. ${ }^{\dagger}$

In order to argue for the above claim, I present two arguments, the "Hole Argument Against Everything" in $\S 3$ and the "Hole-Hole Argument" in $\S 5$. In $\S 4$, I provide important caveats and explain in greater detail some of the concepts I employ in $\S 3$ and $\S 5$. And finally in $\S 6$, I locate my project within the broader post-Hole Argument literature and respond to a challenge raised by Brighouse [5]. Though the arguments of this paper are explicitly aimed at the Hole Argument and diffeomorphism invariance, the concerns they raise are more generally relevant in working out the relationship between generic gauge invariances and the nature of determinism. The position on the Hole Argument which I defend is an instance of "sophisticated determinism".

\section{The Hole Argument Against Spacetime Points}

The Hole Argument, as formulated in [11], is used to argue against the substantivalist view of spacetime. ${ }^{1}$ For the uninitiated, I will very briefly retell this tale and then will show how to widen its result so that everything falls into it. This widening and falling, I use as a reductio against the form of the argument. The Hole Argument begins by first considering an open region of spacetime (the hole). One then takes advantage of the invariant nature of general relativity (GR) under diffemorphisms by shifting all the objects within the hole to new spacetime locations also within the hole. Such a shifting means that some trajectories which had passed through the spacetime points

\footnotetext{
${ }^{\dagger}$ I thank Nick Huggett, John Norton, Carl Hoefer, Sam Fletcher and Jim Weatherall, either for comments on earlier drafts of this paper or for a helpful discussion on some of its core ideas. I also thank the participants of the 2015 philosophy of science meeting in Dubrovnik as well as the southern California philosophy of physics group.

${ }^{1}$ See John Norton's review article [22] for a detailed discussion.
} 
$\left(x_{1}, y_{1}, z_{1}\right)_{n}=\left\{\alpha_{1}\right\}_{n}$ now pass through the spacetime points $\left(x_{2}, y_{2}, z_{2}\right)_{m}=\left\{\alpha_{2}\right\}_{m}$. In general, the point location of fields get all mixed up. And yet, all our physically measurable quantities remain the same: the cat on the mat remains on the mat since both the cat and the mat are similarly shifted by diffeomorphisms. ${ }^{2}$ The predictions of GR are independent of substantival localization facts: where on the substantival manifold physical objects are located.

The crux of the argument is that now, supposedly, we have an indeterministic theory. Before entering the hole, we don't know which trajectory we will be on. The physical data available to us is not enough to determine which trajectory through spacetime points we will take upon entering the hole region. Will the new trajectory pass through these points or those? Moreover, while in the region, we gain no help in determining our trajectory since the physics is the same before and after the shifting. If we think that GR is a deterministic theory, then we are in trouble. According to the Hole Argument, GR cannot determine which path we will take due its invariance under diffeomorphisms.

In terms of possible worlds, standing on a Cauchy slice, there are (at least) two possible worlds identical in their past, yet, which differ in their future. One of these possible world has future-trajectories passing through the sets of spacetime points, $\left\{\alpha_{1}\right\}_{n}$ another has future-trajectories passing through distinct sets $\left\{\alpha_{2}\right\}_{m}$, and our physics cannot tell us which world is actual. ${ }^{3}$ Consequently, the future of any "GRpossible" world is underdetermined by its past. The conclusion to which we are led is that we have made a mistake in treating the manifold and its points as being real things.

\footnotetext{
${ }^{2}$ For a precise treatment of this fact, see Earman and Norton [11].

${ }^{3}$ In fact, each non-trivial diffeomorphism generates a new metric and matter fields physically equivalent to the originals; thus, in general, each past will have infinitely many possible futures.
} 
If we deny that it makes sense to localize physical objects with respect to the manifold and insist that it only makes sense to localize physical objects with respect to other physically observable objects, then we won't end up with indeterminism. We were wrong, so the argument goes, to think of our trajectories as passing through certain points of the spacetime manifold. Rather, the only trajectories that exist are "relational trajectories", i.e. trajectories in terms of physical objects or fields and not spacetime points. In terms of relational trajectories, general relativity is deterministic. Before entering the hole, the relational trajectory is determined: we will pass within some distance of some objects. Thus, we can save determinism but at the cost of shaving a little more off of the substantivalist's ontology. Before presenting the Hole Argument Again Everything, it is essential that I first clarify a few ideas and guard against a few reasonable confusions.

First, Earman and Norton do not assume that we must deny substantivalism just because substantivalism entails that GR is indeterministic. Rather, Earman and Norton argue that if the deterministic status of GR is a physical fact to be determined by observation, then our metaphysics should not automatically decide against it. Since substantivalism does automatically decide against determinism, substantivalism is false. In the following, when referring to Earman and Norton's argument against substnativalism, this is the argument I intend.

Second, in order to forestall a potential worry as well as to properly orient the conversation, we ought to note that the indeterminism generated by the diffeomorphism invariance of GR is not merely that the theory cannot determine, decide or predict where on the manifold we will be next. General relativity cannot determine, for any moment of time, where on the manifold any physical object is located, not merely future moments. The upshot of diffeomorphisms in GR is that localizations with respect to the manifold are not physically determinable facts. The predictions 
of GR are independent of any particular localization of objects within the manifold.

This is particularly important if one thinks about determinism in terms of solving some initial value problem in a globally hyperbolic spacetime. It is usually thought that if a theory is deterministic then, provided all the physical data on some initial Cauchy slice, the theory will determine the evolution of this data across all future Cauchy slices. ${ }^{4}$ Another way of phrasing the question which rests at the heart of this paper is "what are the physical data which our deterministic theory must determine?" Are spacetime point locations part of this data even though the predictions of GR are invariant under diffeomorphisms? Do we require GR to determine at which future spacetime-point-locations the cat is, even though GR cannot say at what spacetime points the cat is now or at any moments in the past? My answer is "no".

Since for any initial Cauchy slice, GR is diffeomorphically invariant, spacetimepoint-locations are not relevant data for the initial value problem and consequently are not relevant for assessing whether or not GR is deterministic. In the following section, I will use the Hole Argument Against Everything to argue that we ought to qualify what we require GR to determine in the way I have suggested here. Namely, that GR is required to determine only those future facts to which the theory is sensitive. Or in other words, GR is only required to determine facts which are included in the physical content of the theory. Or, again, GR is only required to determine certain qualitative properties $[3,2,20]$ which, in the words of David Lewis [16], are the "perfectly natural" ones.

In the following section, I will argue that the notion of determinism used in the Hole Argument ought to be modified because, as stated, the form of the original Hole Argument is invalid. I will do this by providing a counter example to the form of the

\footnotetext{
${ }^{4}$ To be sure, initial value problems or "Cauchy problems" in mathematics are not usually stated in terms of determinism but in terms of solving some differential equation.
} 
Hole Argument which, putatively, results in an absurd conclusion. In generating the following reductio, I will argue that far more than spacetime points are in danger of falling into the hole. I will argue that any metaphysical structure (persons, norms, essences, powers, Aristotelian levels of being) is in danger of the hole precisely because our physical theories do not determine metaphysical properties or facts. The effect of this argument, reductio ad absurdum, will be to force us to analyze the nature of determinism and, derivatively, what we take certain physical theories to be about. I will argue that, in order to avoid the counter examples of $\S 3$, we must limit the scope of determinism. However, in limiting determinism to scope over just those facts or properties of the world for which GR is a theory, substantivalism no longer threatens GR with indeterminism. The following section then has the following structure:

(1) The form of the Hole Argument is invalid since everything falls prey to it.

(2) In preventing everything from falling prey to the Hole Argument we must limit determinism to just that which is included in the physical content of GR.

(3) Substantival localization facts do not belong to the physical content of GR.

Therefore:

(4) Substantival localization facts do not threaten GR with indeterminism. 
(5) Since the Hole Argument argues for indeterminism based on substantival localization facts, the Hole Argument is invalid.

I will argue in $\S 5$ that we cannot bite the bullet and accept the reductio of (1), and will argue throughout this paper for premises (2) and (3).

As a final caveat, the following argument is meant to result in a reductio and has been designed to annoy. One ought to feel that an error has been made, because it has! I will use this error to motivate the analysis involved in defending premises (2) and (3).

\section{The Hole Argument Against Everything}

Throughout the remainder of this paper, I will use the terms 'physical', 'physical content' and 'metaphysical' in intuitive though slightly technical senses which I won't fully explain until $\S 4$. I will say that some property or fact is included in the physical content of a theory if and only if that theory constrains or predicts, for at least one moment of time, the precise value of that property or truth value of that fact. If there is no physical theory for which some property or fact is included in its physical content, I will call these properties or facts 'metaphysical'. 5 I realize that these terms have a long and harried history in philosophy, and I do not intend to solve paradoxes or difficulties inherent in these notions. I only want to make their use explicit. As we will see, these concepts are already implicitly at play in the original Hole Argument. (See $\S 4$ for a longer treatment of the concept "physical content" as well as "determinism".)

Given that our scientific theories cannot detect the effects of our overarching

\footnotetext{
${ }^{5}$ If a certain assumption is necessary in order for a physical theory to be conceptually coherent, I will refrain from calling that assumption metaphysical.
} 
metaphysics, our scientific descriptions of the world are thereby invariant under what I will call 'diffeo-metaphysms' (a protologism). I will define a 'diffeo-metaphysm' to be a mapping between possible worlds which differ only with respect to some particular metaphysical facts. Performing a diffeo-metaphysm on a world changes one or more of its metaphysical facts. For instance, one diffeo-metaphysm might rearrange which Aristotelian "levels of being" some particular objects have, whereas another might reassign which angels propel which planets, or which church Father is the patron saint of salt miners. ${ }^{6}$ Interestingly, since substantival localization facts cannot be determined by GR (or any other empirical science), these facts are metaphysical in the sense just defined. Accordingly, a diffeomorphism, which shifts the substantival spacetime locations of objects, is an instance of a diffeo-metaphysm. I will use the form of the Hole Argument and the fact that our physical theories remain invariant across possible worlds related by particular diffeo-metaphysms, to eradicate everything - or, just about everything.

As an example of how to extend the Hole Argument to everything, consider a world like our own but which also contains certain objects which I will call "persons". 7 For the sake of argument, assume that a single person exists at different places and times and does so by having a soul. According to this metaphysics, souls are a kind of non-physical substance which distinguish persons from one another. Persons are embodied souls, where 'body' does not necessarily refer to human bodies. According to this metaphysics, a person follows a particular soul and not the body in which it is housed. In other words, a person may exchange all of her physical properties and retain her identity but cannot replace her soul without ceasing to be the same individual. In fact, under this metaphysics, it does not even make sense to speak of

\footnotetext{
${ }^{6}$ Which so happens to be Saint Cunegunda or 'Kinga'.

${ }^{7}$ Hoefer [14] puts a similar argument to a different use.
} 
a person exchanging their soul. Let us define a "thing" to be the result of stripping a person of its soul and stipulate that a thing becomes a person by gaining a soul. ${ }^{8}$ Here, I have merely provided definitions; we may not think that souls, persons, or things as defined here exist, yet these concepts are familiar and cogent. By cogent, I mean that these concepts are not self-contradictory and that there are logically possible words which contain things and persons as they have been defined here.

It turns out, and not surprisingly, that GR is soul-invariant. We can associate to each thing any soul we wish, and the description of the world given by GR remains the same. In the case of spacetime points, we may have worried that GR was somehow dependent on them since spacetime points are implicitly referred to by the coordinates of GR; however, this is not so for souls. Souls don't show up at all in GR which makes its invariance under soul swapping all the more obvious. If it makes us feel better, we could build an extended phase space for GR in order to explicitly include a degree of freedom for souls, and then demonstrate that the predictions of GR are soul-invariant. However, doing so would not change our argument and the mathematics involved would be troublesome.

Following Earman and Norton, let us consider an open region (the hole) of spacetime and let us perform a diffeo-metaphysm on whatever persons there are in the hole. We will map this set of persons to a new set of persons by exorcising their souls and gluing them onto new things. ${ }^{9}$ Moreover, unlike diffeomorphisms, this mapping of souls need not even be smooth. ${ }^{10}$ As we might expect, such a diffeo-metaphysm introduces indeterminism into GR. Standing on a Cauchy surface outside the open

\footnotetext{
${ }^{8}$ For the sake of the following argument, persons are meant to be three-dimensional and not four.

${ }^{9}$ Note that I am not claiming that a person gets a new soul, for that would be incoherent given our construction. This mapping reassociates souls with things not persons.

${ }^{10} \mathrm{I}$ intend this to be tongue-in-cheek, but perhaps I should explain. In considering diffeo-metaphysms, we are not doing mathematical physics and thus need not be tied down by smoothness requirements.
} 
region, we cannot say what any person will be doing inside of the hole. Frank, the cat on the mat now may still be Frank the cat then, or it may be Frank the dog at the window then and GR cannot tell us which. ${ }^{11}$ GR cannot tell us where, at any future moment, any person is even if we were to specify where all the persons currently are. If we think that physics is deterministic, which we do, then we are in trouble. General relativity cannot determine which path persons will take through the hole due to its invariance under diffeo-metaphysisms. (For related concerns put to different purposes, see $[14,15])$

Since we started this project by following the lead of Earman and Norton, it is only fitting that we finish it as they do: since persons qua embodied souls automatically decide against determinism, persons are not real things. The actual world does not include souls or persons but does include things. In the actual world, GR does determine what trajectories things take inside the hole. GR might not tell us where Frank is at any future moment, but it does tell us where cat-things are. Physics is safe but so much the worse for souls and Frank. Who knew that general relativity and some "unobjectionable" assumptions about determinism would yield such interesting results? If persons could fall prey to the hole, I suspect that no thing, and perhaps nothing, is safe. ${ }^{12}$

In response to the soul-Hole Argument, the suspicion which we all probably share, is not that persons are somehow in danger now whereas before they weren't, but that the form of the Hole Argument does too much. The trouble with the above argument is that it assumes physics is supposed to say something about souls, or that GR is supposed to determine soul-facts. This mistake enters the argument in assuming

\footnotetext{
${ }^{11}$ In other words, the person associated with the cat-object now may still be associated with the cat-object then, or it may be associated with the dog-object at the window then.

${ }^{12}$ See [13] The point is, that which we call a thing is not metaphysically innocent. Metaphysical assumptions go into how we carve up the world.
} 
that, due to its diffeo-metaphysm, GR is indeterministic. This assumption is true only if we define the determinism of GR to include both metaphysical as well as physical facts. ${ }^{13}$

The Hole Argument against persons qua souls assumes a dichotomy analogous to that used by Earman and Norton in the original Hole Argument:

either (a) accept radical indeterminism, or (b) deny substantivalism.

In the case of the soul-Hole Argument, the dichotomy becomes:

either (a) accept radical indeterminism,

or (b) deny persons.

If, as I suspect, we do not think that the truth of GR nor the determinism of physical theories has anything to do with the existence of persons qua embodied souls, then I suggest we reject this dichotomy in favor of option (c): though GR fails to determine soul-facts, GR remains deterministic for determinism does not require GR to determine soul-facts or any other metaphysical property or fact.

If persons have non-physical, non-supervening minds, we do not require GR to determine what hopes and dreams may occupy those minds, rather we require only that it determine in which head or vat the corresponding brain resides. The point of introducing the Hole Argument Against Everything is to draw our attention to this hidden assumption. In order for the original Hole Argument to work, we must first assume that a proper notion of determinism includes the requirement that GR determine substantival-facts. The Hole Argument works only if we first assume that GR ought to determine which exact set of empirically unobservable substantival ${ }^{13}$ This is exactly Brighouse's point [4]. 
points our physical objects pass through. We don't think that the related assumption is true for souls or any other metaphysical (or 'physically-uninteresting') facts, so why do we think that it is true for substantival-facts?

One answer is to note that unlike soul-facts, GR putatively encodes substantivalfacts in virtue of the fact that the models of $\operatorname{GR}\langle\mathcal{M}, T(p), g(p)\rangle$ include fields defined at spacetime points. Given that the form of the Hole Argument seems to result in a reductio, one response is to argue that I have failed to include an essential, though hidden, premise. That premise being that substantival GR is, in fact, a theory whose physical content includes spacetime points and the substantival localization facts related to them. If this is true, then it could be argued that in order for substantival GR to be physically deterministic, it must determine all such facts. In other words, option (c) would not be true for the original Hole Argument. If this is the case, then there is an important disanalogy between the original Hole Argument and my soul-Hole Argument or the more general Hole Argument Against Everything.

The reason for including this essential premise is the fact that spacetime points seem to show up in the models of GR: $\langle\mathcal{M}, T(p), g(p)\rangle$. Yet, is the presence of manifold points $(p)$ or spatial variables of them sufficient for justifying the claim that the physical content of substantival GR includes the substantival point location of objects? In other words, does the mere presence of some variable entail that a theory determines what exactly happens at values of that variable? Would the physical content of GR automatically increase to include soul-facts merely by adding a soul degree of freedom to the mathematics of our theory? The answer to both of these questions is "no".

At the beginning of this section, I offered to formulate an extension of GR with a soul-degree of freedom. Under the extended theory, GR+, the models $\left\langle\mathcal{M}^{\prime}, T(p, s), g(p, s)\right\rangle$, now include fields which are functions of spacetime points as well as a soul-degree 
of freedom $(s)$. There is no need to actually construct such an odd extension to GR since, in order for this extension to be empirically equivalent to the original theory we will need to force the $s$ variables to be pure gauge (just as the spatial variables are). According to this imagined construction, both GR and GR+ have the same empirical content - the new one just has some extra non-empirical baggage. ${ }^{14}$ The point is, even if GR+ did include a soul degrees of freedom, no physicists would fooled into believing that the mere presence of $s$ in the theory's formulation automatically entails that the physical content of GR+ included souls. My contention is that the physical content of a theory cannot be read off of the mathematics of that theory but must be teased out by putting the mathematics of the theory in contact with experiments. ${ }^{15}$

One of the lessons of modern physics is that just because some degrees of freedom show up in our model, this does not automatically entail that they are physical degrees of freedom of the system. Since GR is diffeomorphically invariant, we know that spacetime points, like soul-degrees of freedom, are in fact not physical. At least they are not physical in the sense defined in this paper: there is no moment of time for which the theory makes any predictions which depend on some set of objects being located at the spacetime points $\left\{\alpha_{1}\right\}_{n}$ rather than $\left\{\alpha_{2}\right\}_{m}$ (see $\S 4$ ).

That substnatival localization facts are not included in the physical content of GR should not be surprising given the homogeneity of the substantivalist's manifold, and it certainly would not have surprised Newton who announced the unknowability of absolute space. According to both Newtonian mechanics and general relativity, we cannot trust a naïve inspection of the variables of the theory in order to know

\footnotetext{
${ }^{14}$ Throughout this paper, I have attempted to make use of the term 'physical' and not 'empirical'. I assume, however, that empirical content is sufficient for physical content.

${ }^{15}$ For related remarks see $[14,7]$.
} 
what the physical degrees of freedom are, but we must also take into account the set of invariances enjoyed by the theory.

Since Newtonian mechanics is invariant under Galilean transformations and GR under diffeomorphisms, neither of these theory's predictions is dependent on the spacetime-point-locations of material objects. As a reminder, though diffeomorphisms frustrate our ability to make predictions regarding the future location of objects, they also frustrate our ability to describe, for any moment of time, the absolute location of objects. Though GR and Newtonian mechanics define fields which take values at spacetime points, which exact set of spacetime points is irrelevant, any will do. ${ }^{16}$ Thus, since neither theory "claims" to determine where any particular field-value is located in substantival space, it is not true that GR encodes substantival localization facts. Given the diffeomorphism invariance of GR, we have no more reason to think that GR ought to determine substantival facts than we have reason to suppose that GR ought to determine soul-facts. What exactly GR should determine I cannot say; however, given that neither souls nor substantival locations make any difference to the physically measurable predictions of the theory, neither are included in the physical content of GR and neither are relevant for the physical determinism of GR.

My solution to the reductio generated by the form of the Hole Argument is to deny that the notion of physical determinism requires our physical theories to determine non-physical facts such as soul-facts or substantival-facts, and it seems that Einstein was of a similar opinion. According to Einstein [27, 21], the physical content of GR is fully captured by point-coincidences between physical objects. And, it is this realization which allowed Einstein to move beyond the Hole Argument and

\footnotetext{
${ }^{16}$ Any set of points will do so long as they are appropriately related to some diffeomorphically invariant model of GR.
} 
accept the generally covariant formulation of GR. If the physical content of GR is captured by the point-coincidences between material objects and not also their absolute location within a substantival manifold, then the indeterminism threatened by the Hole Argument can be ignored since the indeterminism is of a non-physical kind. ${ }^{17}$

Both the original Hole Argument and the soul-Hole Argument are instances of the more general Hole Argument Against Everything. The Hole Argument Against Everything can be modified to accommodate any theory about the world which lies outside the scope of GR - our theory of universals, the truth of the Axiom of Choice, what we take to be the distinguishing mark of abstract objects, whether or not there is absolute time in addition to relativistic time, whether there is a "before" the beginning of the physical universe, whether or not skepticism is true, or who is the patron saint of salt miners - each lead to versions of the Hole Argument precisely because GR says nothing about them.

In summary, the form of the Hole Argument does too much in virtue of using an unqualified notion of determinism. If we fail to limit what we require of our deterministic theories, then spacetime points, souls, and most everything else, can be threatened by hole-type arguments for the argument forms are the same. I have argued, in particular, that since the physical content of GR does not include substantival localization facts, our notion of physical determinism ought not require that GR determine them. And if not the original Hole Argument is invalid.

\footnotetext{
${ }^{17}$ Earman [9] concedes to Maudlin that this might be an uninteresting form of indeterminism. I argue further that it is no form of determinism at all.
} 


\section{Interlude}

Sections $\S 1-3$ constitute, what I take to be, the heart of this paper. In $\S 5$, I provide the Hole-Hole Argument to demonstrate that, if we do not limit the scope of determinism, the debate over the substantival-relational nature of spacetime would be rendered meaningless in virtue of committing a category error. In essence, I will argue that one cannot bite the proverbial bullet and allow the Hole Argument Against Everything to swallow whatever it gets its hands on: spacetime points, haecceities, Aristotelian levels of being, etcetera. In $\S 6$, I demonstrate how this paper fits within the broader post-Hole Argument literature and, in particular, its relation to the work of $[5,3,20,15,4]$. In the remainder of this section, I will explain a bit more carefully how I have used the concepts of "physical content" and "determinism".

For the sake of this paper, some property or kind of fact is included within the physical content of some theory if and only if there is at least one moment of time for which the theory describes or makes predictions regarding the value of that property or the truth of that fact. For example, if for some moment of time and some theoretical context, a theory makes predictions regarding the properties $\left\{P_{i}\right\}$, and at another moment of time, the properties $\left\{P_{j}\right\}$, then the properties $\left\{P_{i}\right\}$ and $\left\{P_{j}\right\}$ are included in the physical content of the theory. This theory, then, is said to be deterministic if, for all moments of time, it predicts, rightly or wrongly, the value of the properties $\left\{P_{i}\right\}$ and $\left\{P_{j}\right\}$. While I intend these definitions to be obvious, it is in fact not at all obvious when the definitions apply for it is not always clear which properties do in fact reside within the physical content of a theory.

I will take determinism to be a property that a theory possesses when that theory determines, decides, predicts, for all moments of time, those properties of the world for which that theory is a theory. In other words, a theory is deterministic if it 
determines, decides, or predicts, for all moments, of time those properties of the world which are included in the physical content of the theory. By limiting the notion of determinism to apply to only the physical content of a theory, I intend to make explicit the fact that theories have limited scopes of applicability. Each physical theory is about some things and not about others, and our notion of determinism ought to reflect this limited scope. Consider the following cases as means of making these definitions concrete.

In the case of quantum mechanics $(\mathrm{QM})$, since there are moments of time for which QM predicts or describes the position of electrons, electron-positions are included in the physical content of QM. Among other things, particle-position is what QM is about. However, since QM cannot predict electron positions for all moments of time, the theory fails to be deterministic. In Newtonian mechanics, since there are moments of time for which the theory describes the position of baseballs, such properties are included in the physical content of Newtonian mechanics. Ignoring Earman's "space invaders" [8], since Newtonian mechanics predicts for all moments of time the position of baseballs, the theory is deterministic with respect these properties. Indeed, Newtonian mechanics is deterministic with respect to all properties or facts for which Newtonian mechanics is a theory. As such, Newtonian mechanics is deterministic simplicitor.

In contrast with particle and baseball positions, since there is no moment of time or theoretical context for which either QM or Newtonian mechanics describes or predicts the truth value of the Axiom of Choice, this truth value is not part of either theory's physical content. Thus, when adjudicating whether or not QM or Newtonian mechanics is deterministic, the truth value of the Axiom of Choice is irrelevant. I do not intend any of this to be controversial. I intend only to make explicit and somewhat more precise what we all take for granted when thinking about physical 
determinism.

Now, in order for a theory to be said to describe, determine, or predict the value of some property, it must be the case that the empirical results of the theory uniquely specify the value of that property for at least one moment of time for some possible situation. Thus, according to the empirical resources of the theory, whether or not these resources are practically feasible, if all possible empirical evidence can never discern between some putative property $P$ and its doppelgänger $P^{\prime}$, then neither $P$ nor $P^{\prime}$ are included in the physical content of the theory. And this of course is exactly the situation for substantivalism: none of the predications of GR, for any moment of time, depend on which set of points $\left\{\alpha_{1}\right\}_{n}$ or $\left\{\alpha_{2}\right\}_{m}$ the material of the universe passes through. Thus, substantival localization facts are not included in GR's physical content and are thereby irrelevant for deciding the deterministic status of GR.

This "uniqueness" requirement tightens the definition for belonging to a theory's physical content and brings this notion into alignment with our attitude towards gauge degrees of freedom. Indeed, we tend to treat the $U(1)$ symmetry of QM differently than we treat the quantum observables of the theory. We interpret the latter as representing genuine physical properties and we bicker over the interpretation of the former. The interpretational debate over the $U(1)$ gauge freedom is most interesting under circumstances such as the Bohm-Aharonov effect in which the gauge potential seems to make an actual difference in what is observed. If it was not for this tenuous connection to empirical observations, there would be little reason for interpreting this mathematical gauge structure as being included in the physical content of QM. This is not to say that the gauge degrees of freedom fail to denote anything, I am agnostic on this point. Perhaps gauge degrees of freedom denote metaphysical potentialities or some other physically opaque aspects of reality. 
In defining "physical content" in a manner which rules out gauge degrees of freedom or, more generally, the properties $P$ and $P^{\prime}$, I am adopting a non-trivial stance in the debate over gauge variables. However, the contrary position is rather unappealing for two reasons. In allowing gauge variables to denote physical properties, we would be, in essence, including within the physical content of a theory, properties which make no empirical difference, directly or indirectly, for any situation allowed by the theory. Why believe that a physical theory is about something for which it can say nothing? ${ }^{18}$ Again, I am not saying that gauge variables fail to represent in toto, but merely that whatever they represent should be distinguished from the full blooded physical content of the theory.

In addition, if we allow gauge variables to denote physical content while still requiring that the theory determine this content for all moments of time in order for the theory to be deterministic, we would make determinism impossible for theories with anything like gauge freedom. Since there is no moment of time for which such theories nail down the value of these gauge properties, there is, by definition, at least one moment for which the theory cannot determine precisely the value of these properties. Thus, all such theories are automatically indeterministic. Under this proposal, quantum mechanics would be ruled indeterministic because of its $U(1)$ gauge freedom and Electromagnetism for its gauge potential. If we do not tighten what we mean by "physical content," we force indeterminism on all theories with anything like gauge freedom. And this, of course, is not something that Earman and Norton will countenance.

If the reader will recall (§2), Earman and Norton's argument against substantival

\footnotetext{
${ }^{18}$ In cases where classical gauge degrees of freedom are quantized and then, for whatever reason, become empirically salient, we have moved into a new theoretical context and have to re-evaluate what we take to be physical.
} 
spacetime hinges on the fact that the metaphysics of substantivalism automatically decides against determinism. However, the opposite situation arises if we do not rule out gauge variables and the like from denoting physical structure: indeterminism becomes a matter of definition and not of empirical observation. Allow me six short caveats before moving onto the Hole-Hole Argument.

First, I am not committed to the term 'metaphysics'. It does not matter what we label the set of facts with which physics cannot help. We could just as well label facts of this kind as 'X-facts', or 'physically-uninteresting-facts' or 'whatever else there is'. More often in the Hole Argument literature, the terms 'non-qualitative' or 'haecceistic' $[5,3,2,20,14,15,25]$ are used to denote, roughly, what I am calling metaphysical facts, while 'qualitative' or 'perfectly natural' $[16,25,6]$ are used to denote, roughly, the set of physical properties or facts. I suspect that the terms 'observable' and 'theoretical' do not correspond with my 'physical' and 'metaphysical'. For instance, there are non-observable terms (i.e. theoretical terms like 'electron') which I would still categorize as being physical since our physical theories constrain their properties. I have opted not to use the terms 'qualitative', 'haecceistic', or 'perfectly natural' since their inclusion would only add an additional layer of obfuscation. For the technical sense of these terms, see the cited works.

Second, I assume that what counts as physical and what counts as metaphysical is decided by some thoroughly theory-ladened process. As the history of science demonstrates, our acceptance of some set of theories and their interpretations constrains, to a large extent, what counts as physical and metaphysical. Though the concept of "physical content" is vague and paradigm dependent, we regularly and successfully rely on it and have a good, though tacit, handle on it. For example, we all agree that the taxonomy of fungi and not Aristotelian "levels of being" lay within the scope of biology, that the ideal gas law is about moles of particles and not of 
the burrowing mammal, and that the content of modern cosmology is not same as ancient Egyptian cosmology. Our agreement on these points indicates that we share some core understanding of what "physical content" amounts to, even if we cannot fully explicate it.

Third, part of what makes the division between the physical and metaphysical aspects of reality theory-ladened arises from the uncertainty over how to interpret gauge degrees of freedom or spaces of transformations under which a theory is invariant. Newtonian mechanics is invariant under Galilean transformations which entail that the theory cannot answer such questions as "where is the Earth absolutely located in substantival space?" Though, Newtonian mechanics can answer other kinds of substantival questions such as "does the Earth move in an absolutely (substantival) straight or curved line?"19 According to the terms defined in this paper, from the perspective of a substantival interpretation of Newtonian mechanics, the first question corresponds to a metaphysical fact while the second, to a physical fact. ${ }^{20}$ This stands in contrast with Aristotelian cosmology according to which space is not homogenous or isotropic; consequently, the absolute location of an object does affect the physical character and potential dynamics of the object. In contrast with Newton then, the absolute location, whether it be superlunar or sublunar, is a physical fact on the Aristotelian theory, with empirically distinct consequences. Here "lunar" is not meant to indicate the moon but the absolute boundary between elements composed of quintessence and those composed of water, earth, air and fire. ${ }^{21}$ Thus,

\footnotetext{
${ }^{19}$ This situation holds for general relativity as well. The theory cannot determine for any past, present or future moment where any object is located but it can determine whether or not it moves along a geodesic (straight line).

${ }^{20}$ Under a relational interpretation, neither question is well defined since they make reference to objects which do not exist.

${ }^{21}$ These consequences will turn out to be false in the long run as orbiting objects eventually plummet to the Earth.
} 
whether or not a certain fact counts as metaphysical or physical depends on the overarching theoretical context in which the fact is being asserted. The distinction is theory-ladened.

Fourth, one must not worry that if determinism requires only that theories determine the physical facts which lie in their scope that determinism will be automatically satisfied. In order to see that this fear is misplaced, consider our paradigmatic indeterministic theory - quantum mechanics. Quantum mechanics stipulates that observables encode physical content and yet the theory is explicitly indeterministic with respect to this content. And, important for the purposes of this paper, QM is not indeterministic because of its $U(1)$ gauge freedom.

Fifth, one might dislike the fact that my argument rests on nebulous concepts such as "physical content" and might thereby be biased against it. I share this concern. However, any defense from the Hole Argument Against Everything must say, in some form or another, that GR is not about "everything" and is only about spacetime points and matter fields, and likewise any response to the soul-hole argument must claim that GR is not about souls and in so doing, both responses have already implicitly made use of "physical content" in claiming what GR is "about".

Sixth, though the arguments I have presented here are able to save the substantivalist from the Hole Argument, this does not entail that the substantivalist is off the philosophical hook. As this conversation and those like it make abundantly clear, the substantivalist is committed to properties or facts which are not physical. We might wonder how one can be justified in believing in the existence of something which we can not measure or otherwise detect? This positivistic concern is an old bedfellow but, strictly speaking, it is not the Hole Argument. And besides, we cannot do away with all metaphysics for, as I will argue in $\S 5$, in order to get the Hole Argument off the ground, we have to first assume that spacetime has a very particular modal 
property which we also cannot measure or otherwise detect.

\section{A Hole Argument Against The Hole Argument: the Hole-Hole Argument}

This section addresses the reader whose reaction to the soul-Hole Argument is to accept the putative reductio. There is no strict contradiction in inferring from the soul-Hole Argument that general relativity requires the nonexistence of souls on threat of indeterminism. Likewise, one might very well respond to the Hole Argument Against Everything by eradicating from their ontology any and all structures which threaten the determinism of GR: souls, angels, powers, dispositions, universals, and patron saints. In essence, such a response refuses to modify the banal and unqualified notion of determinism used in the original Hole Argument as well as the Hole Argument Against Everything.

My purpose in using the following Hole-Hole Argument is to show that those who would wield the Hole Argument must first be committed to the existence of at least one non-qualitative or metaphysical property of spacetime. Namely, that spacetime is the sort of thing for which it is possible to be either substantival or relational. By entering into the substantival-relational debate, we implicitly assume that there is at least one logically possible world wherein spacetime is substantival and another wherein it is relational. Equivalently, in debating between substantival and relational ontologies of spacetime, we have to first assume that we have correctly categorized spacetime as the sort of thing which can be substantival or relational. My claim is not that there is something wrong with this assumption but merely that such modal (or categorical) properties are metaphysical from the standpoint of GR. 
The trouble enters here: if we fail to qualify the notion of determinism used in building hole-type arguments, then there is a hole-type argument which utilizes the above modal assumption to demonstrate the meaninglessness of the substantivalrelational debate. Thus, either the substantival-relational debate is meaningless, and with it the original Hole Argument, or the notion of determinism employed must be modified. At the very least, the notion of determinism ought to be modified so as not to require GR to determine the modal properties of spacetime.

The following Hole-Hole Argument is slightly more abstract than the previous Hole Argument Against Everything, though the form of the argument is the same. The increase in abstraction comes not from the logic of the argument since, in form, it is the same argument, but rather arises from the kind of objects being considered. In the Hole Argument against persons, the relevant objects and properties are persons and what souls are associated with what things. In the following Hole-Hole Argument, the object is spacetime itself and the relevant property is modal in nature; we will assume that spacetime is possibly substantival and possibly relational.

Consider the following two possible worlds, $W_{s}$ and $W_{r}$, which are physically - as I have defined this term - identical to each other and to the actual world. Included in the ontology of $W_{s}$ is a substantival spacetime manifold in which all physical objects are located; whereas in $W_{r}$, there is no substantival spacetime manifold. Perhaps spacetime, in $W_{r}$, is merely a relational structure defined to hold between the material objects of the world be these particles, fields, or some as yet undiscovered fundamental objects. In order to streamline the following discussion, I will describe the non-substantival option as being relational, though this might not be the only non-substantival choice. To be clear then, there are no physical differences between $W_{s}, W_{r}$ and the actual world. None of our physical theories make predications which turn on the difference between $W_{s}$ and $W_{r}$, none of our physical theories contain 
physical laws which decide between $W_{s}$ and $W_{r}$, and we certainly do not have any instruments which can detect whether or not there is, in fact, a substantival manifold.

To reiterate, in considering the Hole Argument to begin with, we assume, in the actual world, that there are two distinct possible worlds, $W_{s}$ and $W_{r}$, one of which is in fact the actual world. ${ }^{22}$ If this assumption is true, I will say that the actual world has property $\mathfrak{P}$. In the first place, $\mathfrak{P}$ tells us something about a modal-access relation which holds from the actual world to some possible worlds. If the actual world has $\mathfrak{P}$, then $W_{s}$ and $W_{r}$ are logically possible with respect to it. In the second place, $\mathfrak{P}$ tells us that one of these possible worlds is the actual world. I realize that $\mathfrak{P}$ is an unnatural way of expressing the assumption of the substantival-relational debate, but it is, in fact, what we assume and will play an important role later in this argument.

Given that the actual world and each of the $W$-worlds are observationally identical - in the way that was explained in the previous section - any GR model of the actual world is a GR model of these possible worlds. In other words $W_{r}$ and $W_{s}$ are equally modeled by the same ordered triplet $\langle\mathcal{M}, T, g\rangle$. In accordance with holetype arguments, we ask: can GR determine, of the actual world, whether or not the future will include a substantival spatial structure or whether the future will include a relational spatial structure? In other words, is the future included in $W_{r}$ or $W_{s}$ ?

Such a question should strike us as odd. Why would we ever expect GR to answer such a strange, erudite, and metaphysical question? And, this is exactly the right response. However, I claim that this is the response we should have to the original Hole Argument (see option (c) in $\S 3$ ), and I will return to this response later.

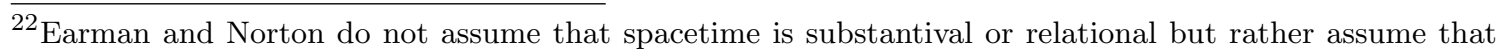
spacetime is either substantival or not substantival. This difference will not make a difference in what follows.
} 
As things stand, there are two possible futures, one relational and the other substantival and since these two possibilities pick out the exact same models in GR, GR does not determine which is actual. Thus, if the actual world has property $\mathfrak{P}$, then GR is indeterministic. Now, one might object that, given a full set of metaphysical facts, we philosophers could indeed decide whether or not the future is substantival. The intuition is that if the initial data of some Cauchy slice includes the fact that the spatial structure is substantival, then "metaphysical consistency" requires that all future slices are also substantival. There are some subtleties in this objection and alternate ways of getting around it but, for the time being, I will simply note that the task is not what "metaphysical consistency" requires but what our physical theory can determine. GR cannot determine whether future spatial regions have a substantival or relational structure.

We might respond to the failure of GR to determine these erudite facts in one of the following three ways:

(a) Accept radical indeterminism.

(b) Bite the proverbial bullet: following the lead of Earman and Norton, we need to give up whatever background metaphysical commitment is responsible for generating the plurality of possible futures and is thereby responsible for the indeterminism of GR.

(c) Qualify 'determinism': in order for a physical theory to be deterministic it need only determine the physical facts within the theory's scope and not also metaphysical facts which lie outside that scope. (See option (c) in $\S 3$.

My suggestion, as always, is that we opt for (c) but in order to see this, let us first explore option (b). 
According to the Hole-Hole Argument and option (b), we ought to deny that the actual world has property $\mathfrak{P}$. Hence, we were wrong in thinking that $W_{s}$ and $W_{r}$ were possible worlds. However, if we deny that the actual world could be either $W_{s}$ or $W_{r}$, then the debate over the substantival vs. relational structure of spacetime is confused. Just as it would be a category mistake to argue over the I.Q. of opaque glass or the flavor of $\pi$, so also it would be a category mistake to argue over the substantival-relational nature of spacetime. If the Hole-Hole Argument is correct and we pursue option (b), then the original Hole Argument, literally, does not make any sense. However, if we think that the original Hole Argument does makes sense and that spacetime is the sort of thing that might be substantival or relational, then I suggest that we pursue option (c).

According to option (c), we must limit what we require of deterministic theories. Do we require GR to determine whether or not certain tiny regions of spacetime are substantival in order for the theory to be deterministic? No. Likewise, if the actual world were substantival, would we require GR to determine the spacetimepoint-location of physical objects? Presumably not. The point is, as soon as we start debating what we mean by 'deterministic physical theory', the substantivalist has an avenue for defending herself against the original Hole Argument; namely, she can simply claim that since spacetime-point-locations make no physical difference to the predictions of any physical theory, these facts are metaphysical in nature and not under the purview of what we require physical theories to determine.

\section{The post-Hole Argument Literature}

In this section, I will locate my project within the broader post-Hole Argument literature and will respond to an important objection from Brighouse [5]. It turns 
out that the Hole-Argument Against Everything can be reconstructed from a combination of positions held by Horwich and Brighouse. Horwich [15] argues that the same trouble facing spacetime points, also faces electrons and other objects as well. Roughly, Horwich asks us to consider two identical worlds which differ only in terms of which electrons are where. In world one, electron A is here and electron B is there. While in in world two electron, A is there and electron B is here. Since these worlds are qualitatively identical, our physical theories are invariant under a swapping of electrons. Thus, our physical laws do not uniquely determine what the future will be like.

Should we conclude then that electrons are somehow illusory? Horwich says "no", and uses the qualitative similarity between world one and two to argue that they are actually the same world. Similarly, we can adapt Horwich's analysis to the case of the Hole Argument and argue that all diffeomorphically related "holeworlds" are merely different descriptions of the same world. We can avoid the terror of the hole and maintain an unqualified notion of determinism by shrinking the space of possible worlds from many to one. If there is only one possible world described by diffeomorphisms, then the future of any Cauchy slice cannot fail to be deterministic. The spirit of this response is codified in [6] as well as in the the sophisticated substantivalist position championed by Hoefer [14]. The much noted downside to this response is that it cannot accommodate the substantialist's intuition, which Earman and Norton [11] refer to as the acid test of substantivalism: "shifted worlds" represent different possibilities. This is not to say that the view is wrong, but if one takes Earman and Norton's acid test seriously, sophisticated substantivalism is no longer an option.

In [4] Brighouse argues that all we require of our physical theories is that they physically determine the future given the past. Facts such as which points on the 
manifold are occupied by which objects, are not physical facts, according to her, and thus are not determined by our physical theories. Brighouse's position $[3,4]$ and my own are identical in this regard and are instances of what Brighouse [5] calls "sophisticated determinism". A benefit of this position is that it naturally accommodates the substantivalist's conviction that shifted worlds represent genuinely distinct possible worlds, though, admittedly, worlds which differ only in terms of "metaphysical" properties. While Brighouse and I share the same conclusion, we differ in how we come to it. In particular, Brighouse does not argue for her conclusion using the Hole Argument Against Everything or the Hole-Hole Argument, as I have done here. As far as I am aware, these two arguments are the only arguments in the literature which have been provided in defense of sophisticated determinism. One can reconstruct my position as a combination of Horwich's and Brighouse's: I argue along with Horwich that many things are are threatened by hole-type arguments and conclude, along with Brighouse, that hole arguments wrongly use unqualified notions of determinism.

Three main lines of response developed shortly after Earman and Norton's paper: metric essentialism [18], sophisticated substantivalism [14, 6, 17, 26], and sophisticated determinism $[3,20,4,25] .{ }^{23}$ The solutions provided by the metric essentialist and the sophisticated substantivalist are tangential to those provided by the sophisticated determinist. One can believe that the metric is essential for the identity of spacetime, or that spacetime points fail to have primitive identities and still agree with a sophisticated understanding of determinism. Admittedly though, introducing a sophisticated notion of determinism will lessen the need for an alternative solution to the Hole Argument.

\footnotetext{
${ }^{23}$ Along with [24], I am using the term 'sophisticated substantivalism' more narrowly than [10] intended when they coined the expression.
} 
In addition to either sophisticated substantivalism or metric essentialism, there are "technical" solutions to the Hole Argument which are also tangential to sophisticated determinism. Two recent examples come to mind. Jim Weatherall [28] implicitly accepts the putative conflict between determinism and substantivalism and proposes a conceptually sophisticated workaround. Weatherall claims that, in deriving the Hole Argument, we have to make certain additional and mistaken assumptions regarding the identity of mathematical points across diffeomorphically related models. And according to Curiel [7], one ought reject the Hole Argument not because it abuses the notion of determinism but since "a proper understanding of diffeomorphism invariance and the way to properly implement it as a formal procedure vitiates [the argument]" (p.10). The details of either of these projects are beside the point since neither Curiel nor Weatherall critique the notion of determinism used in the Hole Argument, let alone raise the issue. ${ }^{24}$ Strictly speaking, these projects are tangential to the sophisticated determinism since they allow an assumption which the sophisticated determinist explicitly rejects.

There remains one final task before concluding. In [5] Brighouse argues against her original $[3,4]$ defenses of sophisticated determinism as well as against Melia's [20] variant of it. Allow me a few words to address her concerns. In that paper, Brighouse insists on the following intuitive, though unqualified, concept of determinism:

I am going to argue that sophisticated determinism ends up violating the spirit of the intuitive conception of determinism that we started with in Section 2, namely, that a world is deterministic if and only if there is at most one possible future compatible with its past. (p.165)

Accordingly, Brighouse lays down the following challenge:

${ }^{24}$ From what I can determine, the last paper to discuss sophisticated determinism is Brighouse [5]. 
Should someone mount an objection against the intuitive conception of determinism, and argue that sometimes, even when there is more than one distinct future compatible with the past then determinism sometimes still reigns? Only if one has provided a criterion for when different futures should count as determinism violating futures and when they should not, and one has provided a justification for this criterion. (p.171)

This seems like a sensible request especially given that the "intuitive notion" actual happens to be intuitive. Unfortunately, as we all know, intuition is often mistaken. I argued in $\S 3$ that if we do not modify the intuitive conception of determinism, then every metaphysical structure, every structure whose properties are not constrained by our physics, is threatened by some hole-type argument and, in $\S 5$, that a nonmodified conception of determinism threatens the meaningfulness of the substantivalrelational debate. Even without a better alternative, we have good reason to abandon the "intuitive notion," for there is no sense in latching oneself to a sinking ship. Fortunately though, I have suggested how we might upgrade the intuitive notion in terms of physical content.

\section{Conclusion}

The Hole Argument is presented as a challenge from GR to substantival interpretations of spacetime. It is claimed that GR challenges such interpretations in virtue of its models being diffeomorphically invariant. In $\S 3$, I used the form of the Hole Argument to demonstrate that GR also challenges the existence of persons (qua embodied souls) in virtue of being invariant under diffeo-metaphysms. In fact, were the form of the Hole Argument valid, everything would fall prey to some holetype argument. In addition, as demonstrated in $\S 5$, the very meaningfulness of the 
substantival-relational debate is lost if the form of the Hole-Argument is valid. Thus, either determinism requires both that there be no metaphysical truths and that the substantival-relational debate is without meaning or, the näive conception of determinism ought to be rejected in favor of some sophisticated variant of it. According to the sophisticated variant which I have outlined, deterministic physical theories are required to determine only physical facts and not also whatever non-physical facts might be true of the world. Which facts are physical and which are not is decided only from within some theory-ladened paradigm. As a final reminder, the central motivation which generated the arguments of this paper is that deterministic theories ought not be required to determine for future moments what they cannot determine for any present or past moments. 


\section{References}

[1] H.G. Alexander. The Leibniz-Clarke Correspondence. Manchester University Press, 1956.

[2] Gordon Belot. New work for counterpart theorists: Determinism. British Journal for the Philosophy of Science, 46(2):185-195, 1995.

[3] Carolyn Brighouse. Spacetime and Holes. In PSA: Proceedings of the Biennial Meeting of the Philosophy of Science Association, pages 117-125. JSTOR, 1994.

[4] Carolyn Brighouse. Determinism and Modality. The British Journal for the Philosophy of Science, 48(4):465-481, 1997.

[5] Carolyn Brighouse. Understanding determinism. In Dennis Dieks, editor, The Understanding of Spacetime II, pages 153-174. Elsevier, 2008.

[6] Jeremy Butterfield. The hole truth. British Journal for the Philosophy of Science, 40(1):1-28, 1989.

[7] Erik Curiel. On the existence of spacetime structure. British Journal for the Philosophy of Science, 2016.

[8] John Earman. A Primer on Determinism. Springer, 1986.

[9] John Earman. Thoroughly Modern McTaggart: or, what McTaggart would have said if he had read the general theory of relativity. Ann Arbor, MI: Michigan Publishing, University of Michigan Library, 2002.

[10] John Earman and Gordon Belot. Pre-socratic quantum gravity. In Physics Meets Philosophy at the Planck Scale. Cambridge University Press, 2001. 
[11] John Earman and John Norton. What Price Spacetime Substantivalism? The Hole Story. British Journal for the Philosophy of Science, pages 515-525, 1987.

[12] Albert Einstein. The foundations of the general relativity. In The Collected Papers of Albert Einstein: Volumen 6, page 153. Princeton University Press, 1997.

[13] Martin Heidegger, William Baynard Barton, Vera Deutsch, and Eugene T Gendlin. What is a Thing? H. Regnery Company, 1967.

[14] Carl Hoefer. Metaphysics of spacetime substantivalism. The Journal of Philosophy, 93(1):5-27, 1996.

[15] Paul Horwich. On the Existence of Time, Space and Space-time. Nous, pages 397-419, 1978.

[16] David Lewis. New work for a theory of universals. Australasian Journal of Philosophy, 61(4):343-377, 1983.

[17] Anna Maidens. Review of john earman's world enough and space-time. British Journal for the Philosophy of Science, 43:129-136, 1992.

[18] Tim Maudlin. The essence of space-time. Proceedings of the Biennial Meeting of the Philosophy of Science Association, 2:82-91, 1988.

[19] Tim Maudlin. Thoroughly Muddled Mctaggart: Or, how to abuse gauge freedom to create metaphysical monostrosities. Ann Arbor, MI: Michigan Publishing, University of Michigan Library, 2002.

[20] Joseph Melia. Holes, haecceitism and two conceptions of determinism. British Journal for the Philosophy of Science, 50(639-664), 1999. 
[21] John D. Norton. General covariance and the foundations of general relativity: Eight decades of dispute. Reports on Progress in Physics, 56(7):791-858, 1993.

[22] John D. Norton. The Hole Argument. In Edward N. Zalta, editor, The Stanford Encyclopedia of Philosophy. SEP, spring 2014 edition, 2014.

[23] Derek Parfit. Reasons and Persons. Oxford University Press, 1984.

[24] Dean Rickles. Symmetry, Structure, and Spacetime. Elsevier, 2007.

[25] Brian Skow. Once upon a spacetime. PhD thesis, New York University, 2005.

[26] John Stachel. Substances, modality and spacetime. Unpublished.

[27] John Stachel. Einstein's search for general covariance. In Einstein and the History of General Relativity (Einstein Studies, Volume 1). Birkhäuser, 1980 (1989).

[28] James Weatherall. Regarding the 'hole argument'. British Journal for the Philosophy of Science, 2016. 